ORIGINAL ARTICLE

\title{
Stromal nitric oxide synthase (NOS) expression correlates with the grade of mammary phyllodes tumour
}

\author{
G M K Tse, F C Wong, A K H Tsang, C S Lee, P C W Lui, A W I Lo, B K B Law, R A Scolyer, R Z Karim, \\ T C Putti
}

See end of article for authors' affiliations

......................

Correspondence to: Dr G M Tse, Department of Anatomical and Cellular Pathology, Prince of Wales Hospital, Ngan Shing Street, Shatin, NT, Hong

Kong, China; garytse@ cuhk.edu.hk

Accepted for publication 13 December 2004
Background: Nitric oxide synthase (NOS), particularly endothelial and inducible forms (e/i-NOS), are expressed in various cancers, including breast cancer. In mammary fibroepithelial lesions, NOS expression in stromal cells has been reported to be lower in fibroadenomas than in phyllodes tumours. Aims: To investigate NOS expression in phyllodes tumours of varying degrees of malignancy. Methods: One hundred and sixty seven mammary phyllodes tumours (97 benign, 47 borderline malignant, and 23 frankly malignant) were evaluated for e-NOS and i-NOS expression by immunohistochemistry. Correlations with previously reported expression of stromal vascular growth factor (VEGF) and microvessel density were also performed.

Results: Stromal expression of e-NOS was absent, weak, moderate, and strong in $43 \%, 31 \%, 13 \%$, and $13 \%$ of benign tumours; $17 \%, 26 \%, 13 \%$, and $44 \%$ of borderline malignant tumours; and $17 \%, 35 \%, 13 \%$, and $35 \%$ of frankly malignant tumours, respectively. Stromal expression of i-NOS was $77 \%, 18 \%, 4 \%$, and $1 \%$ in benign tumours; $42 \%, 28 \%, 19 \%$, and $11 \%$ in borderline malignant tumours; and $43 \%, 13 \%, 26 \%$, and $18 \%$ in frankly malignant tumours, respectively. Stromal expression of both i-NOS and e-NOS was significantly different between the benign and malignant (borderline and frank) groups of phyllodes tumours $(p<0.0001)$. Furthermore, the expression of $i-N O S$ correlated with stromal VEGF expression and microvessel density. The expression of NOS in the epithelial cells was strong, and showed no differences between the different groups of tumours.

Conclusions: Higher stromal expression of NOS in phyllodes tumours is associated with malignancy, suggesting a possible role in malignant progression, particularly metastasising potential.
$\mathrm{N}$ itric oxide (NO) is a free radical gas synthesised by a family of isoenzymes called NO synthases (NOS). ${ }^{12}$ NO acts as an intracellular secondary messenger in various pathways, including those involved in vasodilation, neuronal transmission, and cytotoxicity of inflammatory cells. $^{3}$ The NOS isoenzymes are classified into two groups, namely: (1) the calcium dependent, constitutively expressed constitutive NOS enzymes, which include the endothelial NOS (e-NOS) and the neuronal NOS forms; and (2) calcium independent inducible NOS (i-NOS), which is involved mainly in tumour induced immunosuppression and macrophage mediated cytotoxicity. ${ }^{4}$ The role of NO in tumour biology is still poorly understood, with many authors reporting that NO has both protumour and antitumour effects. ${ }^{5}$ Its protumour effects include promotion of DNA strand breakages ${ }^{6}{ }^{6}$ impairment of the function of the tumour suppressor $\mathrm{p} 53,{ }^{7}$ promotion of neovascularisation or enhancement of blood flow, ${ }^{48}$ and the development of metastases. ${ }^{9}$ NO can also have antitumour effects, ${ }^{10}{ }^{11}$ and this may or may not be related to the observed effect of inducing apoptosis in tumour cells. ${ }^{12}$

"The role of nitric oxide in tumour biology is still poorly understood, with many authors reporting that it has both protumour and antitumour effects"

NOS activity has been demonstrated in a variety of human malignancies, including ovarian cancer, ${ }^{13}$ gastric cancer, ${ }^{14}$ colonic adenoma, ${ }^{15}$ brain tumours, ${ }^{16}{ }^{17}$ head and neck squamous cell carcinoma, ${ }^{18}$ lung tumours, ${ }^{19}{ }^{20}$ prostate carcinoma, ${ }^{21}$ urinary bladder transitional cell carcinoma, ${ }^{22}$ oesophageal adenocarcinoma, ${ }^{23}$ and Kaposi's sarcoma. ${ }^{24}$
In breast cancer, NOS expression and its correlation with various parameters including stage, grade, metastasis status, and precursor lesions has been the subject of much investigation, but with conflicting results. Most studies have concentrated on i-NOS, with smaller numbers reporting on the relation between breast lesions and e-NOS. Many authors have found e-NOS expression to be present in breast carcinoma, ${ }^{352526}$ and its expression has been variably reported as reduced ${ }^{5}$ or increased ${ }^{3}$ compared with normal breast epithelium. It has also been reported that e-NOS expression is variably related to tumour grade, ranging from no correlation ${ }^{26}$ to an inverse correlation with tumour grade (higher expression in lower grade cancers). ${ }^{325}$ Other authors have only detected e-NOS in metaplastic apocrine cells of the breast and not in carcinomas. ${ }^{27}$ The presence of e-NOS positive vessels in the peritumorous tissue but not within the tumour has also been observed..$^{28}$

Expression of i-NOS in breast cancer has been more extensively studied. Most authors have found increased expression of i-NOS in breast cancers, ${ }^{3}{ }^{30-33}$ compared with weak or absent expression seen in benign breast epithelium; others have found decreased expression compared with benign breast epithelium. ${ }^{528}$ Among those studies showing increased i-NOS expression in breast cancers, some found a correlation between increased expression and tumour grade (higher grade, higher expression), ${ }^{3} 303233$ whereas others demonstrated an inverse relation (higher grade, lower expression). ${ }^{31}$ In addition, i-NOS expression was thought to correlate with metastasis development in some studies. ${ }^{94}$

Abbreviations: e/i-NOS, endothelial/inducible nitric oxide synthase; NO, nitric oxide; VEGF, vascular endothelial growth factor 
Very few studies have documented the expression of NOS in fibroepithelial lesions of the breast. In one report, negative i-NOS expression was noted in four cases of fibroadenoma. ${ }^{27}$ Another report noted the presence of nitrate and nitrite but negative NOS activity in cell culture of a single case of phyllodes tumour of unknown malignant potential. ${ }^{33}$ The largest study of mammary fibroepithelial lesions reported in the literature so far found that i-NOS expression was higher in epithelial cells and lower in stromal cells of fibroadenomas compared with phyllodes tumours. ${ }^{35}$

Mammary phyllodes tumour is an uncommon stromal epithelial neoplasm that usually occurs in middle aged women. ${ }^{36-40}$ Determining its malignant potential is difficult, but based on a combination of histological criteria, phyllodes tumours can be divided into benign, borderline malignant, and frankly malignant groups. ${ }^{36}{ }^{41}$ Although all groups of phyllodes tumours show a propensity to recur locally, the borderline and frankly malignant groups may also metastasise to other visceral organs.

In our study, we investigated the expression of NOS activity, including both endogenous (e-NOS) and inducible (i-NOS) forms, in a large series of mammary phyllodes tumours and assessed its correlation with tumour grade. We also compared the expression of i-NOS with the previous assessment of vascular endothelial growth factor (VEGF) expression and microvessel density in the same group of lesions. ${ }^{42} 43$

\section{MATERIALS AND METHODS}

The histological files of four participating institutions in three different countries were searched for phyllodes tumours of the breast. The number of years searched ranged from four to 15. The paraffin wax blocks were retrieved and $4 \mu \mathrm{m}$ sections were prepared routinely and stained with haematoxylin and eosin. All the slides from the various institutions were collected in one centre, where all the immunohistochemical staining was performed. The haematoxylin and eosin slides were reviewed by one author and the diagnosis was compared with the original one. Discrepant cases $(<5 \%)$ were reassessed together with the submitting pathologist and a consensus diagnosis was made. The following histological parameters were assessed: 1, stromal cellularity; 2, nuclear pleomorphism; 3, stromal overgrowth; 4, mitotic rate; and 5, margin of the tumour (whether infiltrative or rounded). Parameters 1 and 2 were graded as low/mild, moderate, or severe; stromal overgrowth was graded as present or absent; and the mitotic count was expressed as the number of mitotic figures for each 10 high power fields $(\times 400$ magnification; Nikon Labophot, field area $0.19 \mathrm{~mm}^{2}$ ). As described previously, ${ }^{44}$ a diagnosis of benign phyllodes tumour was made when there was low cellularity, no stromal overgrowth, mild pleomorphism, a rounded margin, and a mitotic count of $\leqslant 2 / 10$ high power fields. Malignant phyllodes tumour was diagnosed when the mitotic count was $\geqslant 5 / 10$ high power fields, together with stromal overgrowth and an infiltrative margin (fig 1). Phyllodes tumour of borderline malignancy was diagnosed when the criteria for malignant phyllodes tumour were not totally fulfilled.

For the assessment of e-NOS and i-NOS expression, a representative slide from each case was stained using an antibody against e-NOS and i-NOS (both from Transduction Laboratories, Lexington, Kentucky, USA; 1/200 dilution) with the avidin biotin method and microwave antigen retrieval. Cytoplasmic staining of the epithelial and stromal cells was assessed for intensity (graded as 0 if there was no staining, low, intermediate, or high if the staining was weak, moderate, or strong, respectively; fig 2). For e-NOS, staining of the endothelium was taken as strong positive. The percentage of stained cells was also assessed. The overall

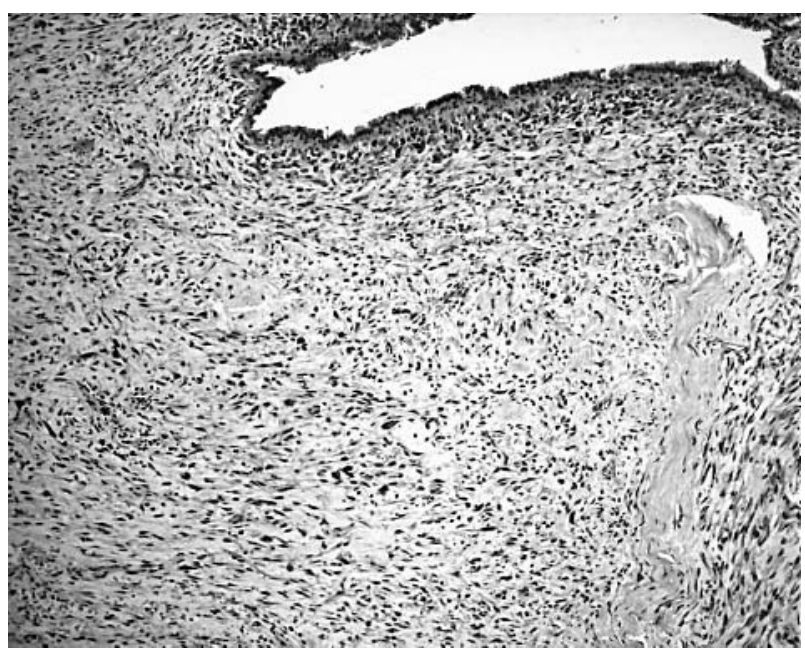

Figure 1 Photomicrograph showing a frankly malignant phyllodes tumour (haematoxylin and eosin staining; original magnification, $\times 100$ ).

staining pattern was considered negative if there was no staining, weak if there was only weak staining, moderate if there was moderate to strong staining in less than $50 \%$ of the cells, and strong if there was moderate to strong staining in $50 \%$ or more of the cells. The slides from each case were assessed by three of the authors from the same centre, and a consensus was reached regarding the staining intensity and percentage of cells stained.

The phyllodes tumours in our series had previously been assessed for VEGF expression by immunohistochemistry and for microvessel density by the hot spot method (applying to CD31 positive structures in the stroma), the results of which have been published..$^{42}$ Because increased vascularity has been cited as one of the purported mechanisms for the actions of i-NOS, ${ }^{48}$ VEGF expression and microvessel density of the stroma were also assessed for a correlation with i-NOS expression.

For statistical analysis, the $\chi^{2}$ test was used to compare patients' ages, tumour size, and e-NOS and i-NOS expression in the epithelial cells and stromal cells between the benign, borderline malignant, and frankly malignant groups of

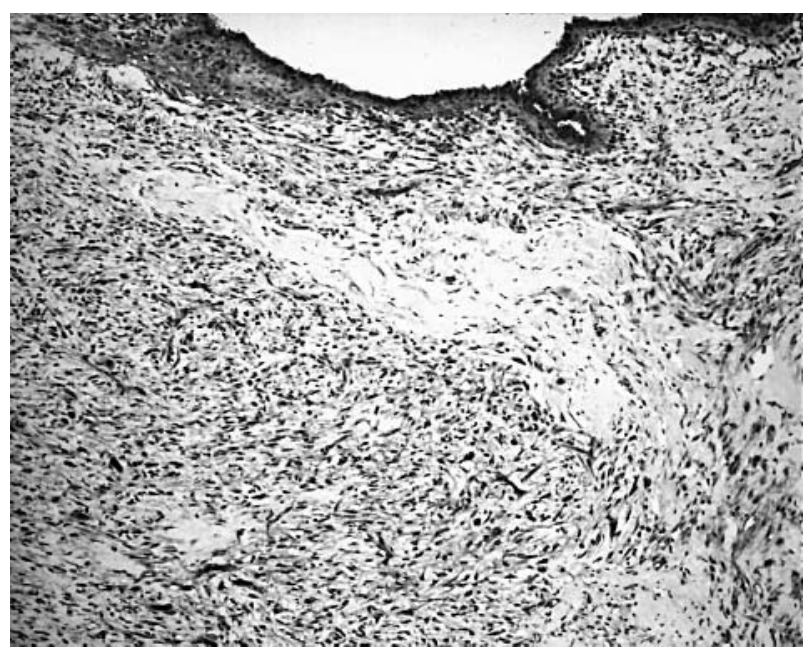

Figure 2 Photomicrograph showing the same frankly malignant phyllodes tumour with strong expression of endothelial nitric oxide synthase in the stromal cells, epithelial cells, and blood vessels (original magnification, $\times 100$ ). 
phyllodes tumours. ANOVA was used to assess the correlation between i-NOS, VEGF expression, and microvessel density of the stroma. Multinominal logistic regression was used to evaluate whether i-NOS, VEGF expression, or microvessel density was a predictor of malignancy of phyllodes tumours. Significance was established at $\mathrm{p}<0.05$.

\section{RESULTS}

In total, 167 mammary phyllodes tumours were retrieved: 97 benign, 47 borderline malignant, and 23 frankly malignant tumours. These tumours were derived from 163 patients, with two tumours (one original and one recurrent) from each of two patients, and the original tumour and two recurrences from one patient. In the remaining 160 tumours, there were 148 tumours that did not recur, three original tumours that later recurred, eight first recurrences, and one second recurrence.

All the patients were female, and included 98 Chinese, 29 whites, 11 Malaysians, seven Indians, and seven other Asians; in 11 patients the ethnicity was not known. The overall age range was $17-77$ years, with a mean of 42 . Eighty one tumours were on the left side, and 85 were on the right. In one tumour, the side was not known. The tumour size range was $0.8-22 \mathrm{~cm}$, with a mean of 4.8 . There was a progressive increase of the patient's age and tumour size from benign to borderline malignant to frankly malignant phyllodes tumours $(\mathrm{p}<0.05)$.

Table 1 shows the overall expression of i-NOS and e-NOS in the entire series, and tables 2 and 3 show the expression of i-NOS and e-NOS among the different categories of phyllodes tumour.

In general, staining for both i-NOS and e-NOS was positive for the epithelial component of all grades of phyllodes tumour, but stromal expression of both forms of NOS increased with increasing grade.

There was no significant difference in the epithelial expression of i-NOS or e-NOS in the entire study population of phyllodes tumours or between tumours of differing degrees of malignancy.

For the stromal expression of both i-NOS and e-NOS, the differences between the benign and borderline malignant groups and between the benign and frankly malignant groups were significant $(p<0.05)$. On further analysis, when the tumours were grouped into two categoriesnamely, benign and malignant (which includes the borderline and frankly malignant subgroups)-the difference between these two groups of tumours became highly significant $(p<0.0001)$. There was no significant difference between these groups for the epithelial component.

In the entire series, there were 13 recurrent phyllodes tumours: five benign, six borderline malignant, and two

Table 1 Expression of i-NOS and e-NOS in the stromal and epithelial components of phyllodes tumours

\begin{tabular}{lll}
\hline NOS isoform expression & Epithelium & Stroma \\
\hline i-NOS & $12(7 \%)$ & $105(63 \%)$ \\
Absent & $58(35 \%)$ & $33(20 \%)$ \\
Weak & $14(8 \%)$ & $19(11 \%)$ \\
Moderate & $83(50 \%)$ & $10(6 \%)$ \\
Strong & $167(100 \%)$ & $167(100 \%)$ \\
Total & $4(2 \%)$ & $53(32 \%)$ \\
e-NOS & $45(28 \%)$ & $50(30 \%)$ \\
Absent & $0(0 \%)$ & $22(13 \%)$ \\
Weak & $118(70 \%)$ & $42(25 \%)$ \\
Moderate & $167(100 \%)$ & $167(100 \%)$ \\
Strong & & \\
Total & & \\
\hline e/i-NOS, endothelial/inducible nitric oxide synthase.
\end{tabular}

Table 2 Expression of inducible nitric oxide synthase (i-NOS) in the epithelium and stroma of benign, borderline, and malignant phyllodes tumours

\begin{tabular}{llll}
\hline i-NOS isoform expression & Benign & Borderline & Malignant \\
\hline Epithelium & $5(5 \%)$ & $2(4 \%)$ & $5(22 \%)$ \\
Absent & $41(42 \%)$ & $13(28 \%)$ & $4(17 \%)$ \\
Weak & $8(8 \%)$ & $3(6 \%)$ & $3(13 \%)$ \\
Moderate & $43(45 \%)$ & $29(62 \%)$ & $11(48 \%)$ \\
Strong & $97(100 \%)$ & $47(100 \%)$ & $23(100 \%)$ \\
Total & $75(77 \%)$ & $20(42 \%)$ & $10(43 \%)$ \\
Stroma & $17(18 \%)$ & $13(28 \%)$ & $3(13 \%)$ \\
Absent & $4(4 \%)$ & $9(19 \%)$ & $6(26 \%)$ \\
Weak & $1(1 \%)$ & $5(11 \%)$ & $4(18 \%)$ \\
Moderate & $97(100 \%)$ & $47(100 \%)$ & $23(100 \%)$ \\
Strong & & & \\
Total & & \\
\hline
\end{tabular}

Table 3 The expression of endothelial nitric oxide synthase (e-NOS) in the epithelium and stroma of benign, borderline, and malignant phyllodes tumours

\begin{tabular}{llll}
\hline e-NOS isoform expression & Benign & Borderline & Malignant \\
\hline Epithelium & $2(2 \%)$ & $0(0 \%)$ & $2(9 \%)$ \\
Absent & $25(26 \%)$ & $11(23 \%)$ & $9(39 \%)$ \\
Weak & $0(0 \%)$ & $0(0 \%)$ & $0(0 \%)$ \\
Moderate & $70(72 \%)$ & $36(77 \%)$ & $12(18 \%)$ \\
Strong & $97(100 \%)$ & $47(100 \%)$ & $23(100 \%)$ \\
Total & $41(43 \%)$ & $8(17 \%)$ & $4(17 \%)$ \\
Stroma & $30(31 \%)$ & $12(26 \%)$ & $8(35 \%)$ \\
Absent & $13(13 \%)$ & $6(13 \%)$ & $3(13 \%)$ \\
Weak & $13(13 \%)$ & $21(44 \%)$ & $8(35 \%)$ \\
Moderate & $97(100 \%)$ & $47(100 \%)$ & $23(100 \%)$ \\
Strong & & & \\
Total & & & \\
\hline
\end{tabular}

frankly malignant tumours. The epithelial and stromal expression of i-NOS and e-NOS showed no correlation with tumour grade.

Previously, we found that the expression of VEGF in the stromal cells of phyllodes tumours was about $30 \%,{ }^{42}$ and the level of expression correlated with the degree of malignancy. Higher levels of VEGF expression were seen in higher grade phyllodes tumours $(\mathrm{p}<0.001)$. A strong correlation between microvessel density and tumour grade was also demonstrated previously $(\mathrm{p}<0.0001) .{ }^{43}$ There was also a strong correlation between stromal VEGF expression and stromal microvessel density $(\mathrm{p}<0.0001){ }^{42}$

The expression of stromal i-NOS correlated with stromal VEGF expression and with stromal microvessel density $(\mathrm{p}<0.001)$. However, using multinominal logistic regression, stromal i-NOS and microvessel density $(p<0.001)$, but not stromal VEGF expression, were predictors of malignancy in phyllodes tumours.

\section{DISCUSSION}

Reports on the activity of NOS in breast cancer have been conflicting, with different or completely opposing findings being reported. Most authors reported the presence of NOS activity in carcinoma. ${ }^{352526}{ }^{30-33}$ Among these, some reported higher NOS activity in malignant than in benign breast tissue, ${ }^{30-33}$ encompassing both a positive correlation between NOS activity and tumour grade $e^{3032} 33$ and a negative correlation, ${ }^{325} 31$ whereas others reported the reverse, ${ }^{528}$ and in benign apocrine metaplasia but not in carcinoma. ${ }^{27}$ These paradoxical results may be explained by the fact that at low 
concentrations, NOS has a tumour promoting effect, whereas at higher concentrations, it has antitumour activity. ${ }^{2}$

Another consistent observation is that NOS appears to correlate with tumour metastasis, ${ }^{94}$ possibly related to increased angiogenesis. ${ }^{32} 34$

In general, for breast cancer there are more reports of tumour cells expressing NOS, both in primary tumours and metastases. Tumour grade appears to be the only tumour parameter that shows a relation with NOS expression. However, there have been conflicting reports on this relation, and further evaluations are needed to resolve the issue.

There have been only rare reports documenting NOS activity in the stroma of breast cancer, and in fibroepithelial lesions such as fibroadenomas and phyllodes tumours. In one interesting and unique study of 111 breast carcinomas, stromal cell expression of i-NOS was detected in all cases, with variable intensity. ${ }^{32}$ In this series, the stromal intensity was related to the intensity of carcinomatous epithelial staining, the presence of metastases, and stromal vascularity. This may suggest a possible interaction between the carcinoma cells and the adjacent stroma, and also supports the contention that NOS expression positively correlates with angiogenesis, thereby facilitating metastasis. In the only study of NOS in phyllodes tumours and fibroadenomas, ${ }^{35}$ Soares et al reported that there was an increased rate of expression of e-NOS in the stroma of phyllodes tumours when compared with fibroadenomas. The results of our study are consistent with the aforementioned series, and furthermore, have shown that i-NOS expression is similarly increased in higher grade lesions.

\section{"Stromal nitric oxide synthase expression may be related} to the ability of the tumour to metastasise"

Because the grading of phyllodes tumours is based on a collective criterion involving several histological parameters, the division into benign, borderline malignant, and frankly malignant can be arbitrary. When the phyllodes tumours are grouped into two categories, taking into consideration the ability to metastasise, they can then be divided into benign and malignant, with this last group encompassing conventional borderline malignant and frankly malignant cases. Under such grouping, the expression of NOS within the stromal cells shows a distinct and highly significant difference, raising the possibility that stromal NOS expression may be related to the ability of the tumour to metastasise. This is in keeping with the notion that NOS facilitates or is associated with metastatic disease development in breast carcinoma.

One of the purported mechanisms of i-NOS activity involves vascular proliferation, and comparison with our previously published results ${ }^{42}{ }^{43}$ showed that there was a strong correlation with both VEGF and microvessel density, raising the possibility that increased vascularity of the lesion may be the effector mechanism for the action of i-NOS.

One of the limitations of our study is that only two cases eventually metastasised, and the number of cases that recurred was also small, making further evaluation of the NOS status of this poor prognostic subgroup impractical.

In summary, our study has demonstrated both epithelial and stromal expression of NOS in phyllodes tumours, and has shown a positive correlation between NOS expression in stromal cells and tumour grade. Significantly higher expression is noted in those tumours with metastatic potential (borderline and frankly malignant phyllodes). This may provide biological evidence to support the histopathological grading system that is commonly used for this rare tumour. ${ }^{36}{ }^{41}$ The correlation between i-NOS expression and VEGF and microvessel density in the stroma may point to
Take home messages

- Nitric oxide synthase (NOS) is expressed in both the epithelial and stromal cells of phyllodes tumours

- There was a positive correlation between NOS expression in stromal cells and tumour grade

- There was a strong correlation between inducible NOS and both VEGF and microvessel density

- Higher stromal expression of NOS in phyllodes tumours was associated with malignancy, suggesting a possible role in malignant progression, particularly metastasising potential

increased vascularity as one of the possible effector mechanisms. Further evaluation of those cases with recurrences or metastases, in particular NOS/VEGF expression and stromal microvessel density, may provide new insights into the pathogenesis and facilitate the prognostication of phyllodes tumours.

\section{Authors' affiliations}

G M K Tse, F C Wong, A K H Tsang, A W I Lo, Department of

Anatomical and Cellular Pathology, Prince of Wales Hospital, Chinese University of Hong Kong, Ngan Shing Street, Shatin, NT, Hong Kong, China

B K B Law, Department of Surgery, Prince of Wales Hospital, Chinese University of Hong Kong

P C W Lui, Department of Pathology, United Christian Hospital, Kwan Tong, Kowloon, Hong Kong, China

T C Putti, Department of Pathology, National University Hospital, 5 Lower Kent Ridge Road, Singapore, 119074

C S Lee, R A Scolyer, R Z Karim, Department of Pathology, University of Sydney, and Department of Anatomical Pathology, Royal Prince Alfred Hospital, Sydney, 2050 Australia

\section{REFERENCES}

1 Knowles RG, Moncada S. Nitric oxide synthases in mammals. Biochem J 1994;298:249-58.

2 Anggard E. Nitric oxide: mediator, murderer, and medicine. Lancet 1994;343:1199-206.

3 Loibl S, von Minckwitz G, Weber S, et al. Expression of endothelial and inducible nitric oxide synthase in benign and malignant lesions of the breast and measurement of nitric oxide using electron paramagnetic resonance spectroscopy. Cancer 2002;95:1191-8.

4 Jenkins DC, Charles IG, Thomsen LL, et al. Roles of nitric oxide in tumor growth. Proc Natl Acad Sci U S A 1995;92:4392-6.

5 Martin JH, Alalami O, van den Berg HW. Reduced expression of endothelial and inducible nitric oxide synthase in a human breast cancer cell line which has acquired estrogen independence. Cancer Lett 1999;144:65-74.

6 Yoshie Y, Ohshima H. Synergistic induction of DNA strand breakage by catechol-estrogen and nitric oxide: implications for hormonal carcinogenesis. Free Radic Biol Med 1998;24:341-8.

7 Calmels S, Hainaut $P$, Ohshima $H$. Nitric oxide induces conformational and functional modifications of wild-type p53 tumor suppressor protein. Cancer Res 1997; 57:3365-9.

8 Andrade SP, Hart IR, Piper PJ. Inhibitors of nitric oxide synthase selectively reduce flow in tumor-associated neovasculature. $\mathrm{Br} J$ Pharmacol 1992;107:1092-5.

9 Duenas-Gonzalez A, Isales CM, del Mar Abad-Hernandez M, et al. Expression of inducible nitric oxide synthase in breast cancer correlates with metastatic disease. Mod Pathol 1997; 10:645-9.

10 Stuehr DJ, Nathan CF. Nitric oxide. A macrophage product responsible for cytostasis and respiratory inhibition in tumor target cells. J Exp Med 1989; 169:1543-55

11 Martin JH, Edwards SW. Changes in mechanisms of monocyte/macrophagemediated cytotoxicity during culture. Reactive oxygen intermediates are involved in monocyte-mediated cytotoxicity, whereas reactive nitrogen intermediates are employed by macrophages in tumor cell killing. J Immunol 1993; 150:3478-86

12 Cui S, Reichner JS, Mateo RB, Albina JE. Activated murine macrophages induce apoptosis in tumor cells through nitric oxide-dependent or -independent mechanisms. Cancer Res 1994;54:2462-7.

13 Thomsen LL, Lawton FG, Knowles RG, et al. Nitric oxide synthase activity in human gynecological cancer. Cancer Res 1994;54:1352-4. 
14 Rajnakova A, Goh PM, Chan ST, et al. Expression of differential nitric oxide synthase isoforms in human normal gastric mucosa and gastric cancer tissue. Carcinogenesis 1997;18:1841-5.

15 Ambs S, Merriam WG, Bennett WP, et al. Frequent nitric oxide synthase-2 expression in human colon adenomas: implication for tumor angiogenesis and colon cancer progression. Cancer Res 2000;58:334-41.

16 Cobbs CS, Brenman JE, Aldape KD, et al. Expression of nitric oxide synthase in human central nervous system tumours. Cancer Res 1995;55:727-30.

17 Hara E, Takahashi K, Tominaga T, et al. Expression of heme oxygenase and inducible nitric oxide synthase mRNA in human brain tumours. Biochem Biophys Res Commun 1996;224:153-8.

18 Rosbe KW, Prazma J, Petrusz P, et al. Immunohistochemical characterization of nitric oxide synthase activity in squamous cell carcinoma of the head and neck. Otolaryngol Head Neck Surg 1995;1 13:541-9.

19 Ambs S, Bennett WP, Merriam WG, et al. Vascular endothelial growth factor and nitric oxide synthase expression in human lung cancer and the relation to p53. Br J Cancer 1998;78:233-9.

20 Fujimoto $\mathbf{H}$, Sasaki J, Matsumoto $M$, et al. Significant correlation of nitric oxide synthase activity and p53 gene mutation in stage I lung adenocarcinoma. Jpn J Cancer Res 1998;89:696-702.

21 Klotz T, Bloch W, Volberg C, et al. Selective expression of inducible nitric oxide synthase in human prostate carcinoma. Cancer 1998;82:1897-903.

22 Swana HS, Smith SD, Perrotta PL, et al. Inducible nitric oxide synthase with transitional cell carcinoma of the bladder. J Urol 1999;161:630-4.

23 Wilson KT, Fu S, Ramanujam KS, et al. Increased expression of inducible nitric oxide synthase and cyclooxygenase- 2 in Barrett's esophagus and associated adenocarcinomas. Cancer Res 1998;58:2929-34.

24 Weninger W, Rendl M, Pammer J, et al. Nitric oxide synthases in Kaposi's sarcoma are expressed predominantly by vessels and tissue macrophages. Lab Invest 1998;78:949-55.

25 Martin JH, Begum S, Alalami O, et al. Endothelial nitric oxide synthase: correlation with histologic grade, lymph node status and estrogen receptor expression in human breast cancer. Tumour Biol 2000;21:90-7.

26 Vakkala M, Paakko P, Soini Y. eNOS expression is associated with the estrogen and progesterone receptor status in invasive breast carcinoma. Int J Oncol 2000;17:667-71

27 Tschugguel W, Knogler W, Czerwenka K, et al. Presence of endothelial calcium-dependent nitric oxide synthase in breast apocrine metaplasia. Br J Cancer 1996;74:1423-6.

28 Tschugguel W, Schneeberger C, Unfried G, et al. Expression of inducible nitric oxide synthase in human breast cancer depends on tumor grade. Breast Cancer Res Treat 1999;56:145-51.
29 Mortensen K, Holck S, Christensen IJ, et al. Endothelial cell nitric oxide synthase in peritumoral microvessels is a favorable prognostic indicator in premenopausal breast cancer patients. Clin Cancer Res 1999;5:1093-7.

30 De Paepe B, Verstraeten VM, De Potter CR, et al. Increased angiotensin II type-2 receptor density in hyperplasia, DCIS and invasive carcinoma of the breast is paralleled with increased iNOS expression. Histochem Cell Biol 2002;117:13-19.

31 Reveneau S, Arnould L, Jolimoy G, et al. Nitric oxide synthase in human breast cancer is associated with tumor grade, proliferation rate, and expression of progesterone receptors. Lab Invest 1999;79:1215-25.

32 Vakkala M, Kahlos K, Lakari E, et al. Inducible nitric oxide synthase expression, apoptosis, and angiogenesis in in situ and invasive breast carcinomas. Clin Cancer Res 2000;6:2408-16.

33 Thomsen LL, Miles DW, Happerfield L, et al. Nitric oxide synthase activity in human breast cancer. Br J Cancer 1995;72:41-4.

34 Brennan PA, Sharma S, Bowden JR, et al. Expression of inducible nitric oxide synthase in bone metastases. Eur J Surg Oncol 2003;29:619-23.

35 Soares FA, Maciel MS, Neto M. Nitric oxide synthases, p53, VEGF and PDGF expression in biphasic tumours of the breast. Histopathology 2002:41(suppl 1):4.

36 Azzopardi JG. Sarcoma of the breast. In: Bennington J, ed. Problems in breast pathology. Vol II, Major problems in pathology. Philadelphia: WB Saunders, 1979:355-9.

37 Palmer ML, De Risi DC, Pelikan A, et al. Treatment options and recurrence potential for cystosarcoma phyllodes. Surg Gynecol Obstet 1990;170:193-6.

38 Kario K, Meada S, Mizuno Y, et al. Phyllodes tumor of the breast: a clinocopathologic study of 34 cases. J Surg Oncol 1990;45:46-51.

39 Rowell MD, Perry RR, Hsiu JG, et al. Phyllodes tumours. Am J Surg 1993:165:376-9.

40 Cole-Beuglet C, Soriano R Kurtz AB, Meyer JE, et al. Ultrasound, X-ray mammography and histopathology of cystosarcoma phyllodes. Radiology 1983; 146:481-6

41 Rosen PP. Breast pathology, 2nd ed. Philadelphia: Lippincott-Raven, 2000:176-97.

42 Tse GM, Lui PC, Lee CS, et al. Stromal expression of vascular endothelial growth factor correlates with tumor grade and microvessel density in mammary phyllodes tumors: a multicenter study of 185 cases. Hum Pathol 2004;35:1053-7.

43 Tse GM, Lui PC, Scolyer RA, et al. Tumour angiogenesis and p53 protein expression in mammary phyllodes tumors. Mod Pathol 2003;16:1007-13.

44 Tse GM, Putti TC, Kung FY, et al. Increased p53 protein expression in malignant mammary phyllodes tumours. Mod Pathol 2002;15:734-40. 